\title{
DIFERENÇAS NA INFESTAÇÃO DE Aphis gossypii EM PLANTAS DE ALGODOEIRO CULTIVAR 'IAC-RM3' TRATADAS COM REGULADORES DE CRESCIMENTO *
}

\author{
Paulo R. C. Castro ** \\ Carros J. Rossetto ***
}

\section{RESUMO}

\begin{abstract}
Estudou-se a influência da aplicação de reguladores de cresci-

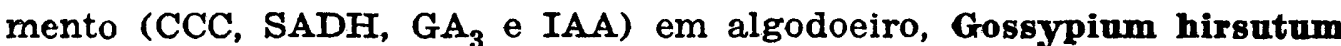
L. cv. 'IAC-RM3', na infestação de Aphis gossypii Glover, 1876; em condições de casa de vegetação.

A realização de duas determinações no nível de infestação dos afídios, evidenciou que plantas tratadas com CCC mostram niveis superiores de infestação com relação às tratadas com $\mathrm{GA}_{3}$ a $100 \mathrm{ppm}$; sendo que a aplicação de SADH a $4000 \mathrm{ppm}$ também promoveu uma tendência de maior infestação com relação ao GA $_{3}$ a $100 \mathrm{ppm}$.

Estes resultados parecem revelar que os retardadores de crescimento promovem um equilíbrio hídrico interno nas plantas mais favorável, mantendo o potencial osmótico mais elevado e possibilitando uma melhor alimentação do afídio. Plantas tratadas com GA a $100 \mathrm{ppm}$ parecem sofrer maiores déficits hídricos, não favorecendo o estabelecimento das colônias, nas condições estudadas.
\end{abstract}

\section{INTRODUÇAOO}

O pulgão do algodoeiro (Aphis gossypii Glover, 1876) é um inseto de tamanho pequeno, com coloração variável do amarelo claro ao verde escuro. Esses afídios vivem sob as folhas e em brotações da planta, sugando a seiva. Inicialmente os indivíduos são ápteros; crescendo a população de maneira muito intensa há falta de alimento, aparecendo então as formas aladas que voam para outras plantas, constituindo novas colônias (GALLO et al., 1970). Os pulgões quando não são controlados, reduzem a produção do algodoeiro em cerca de 44\% (CALCAGNOLO \& SAUER, 1954).

- Entregue para publicação em 29/08/1974.

** Departamento de Botánica. E. S. A. «Luiz de Queiroz» - U. S. P.

*** Seção de Entomologia Fitotécnica. Instituto Agronômico do Estado de São Paulo. Bolsista do CNPq. 
Os afídios estão dentre os insetos fitófagos que mais se adaptam a seus hospedeiros; assim sendo, mesmo pequenas variações morfo-fisiológicas das plantas hospedeiras podem ter efeitos sensíveis nos afídios que as colonizam (VAN EMDEN, 1964). Efeitos do crescimento e senescência vegetal, relações hídricas e resistência varietal, foram descritos por KENNEDY (1958).

LEES (1926) relacionando o ataque de insetos com a condição interna da planta hospedeira, considerou que a irrigação de culturas pode promover um aumento na infestação de afídios com relação às culturas não irrigadas.

Em Couve de Bruxelas, Myzus persicae (Sulzer, 1776) e Brevicoryne brassicae (L., 1758) colonizam paticularmente folhas velhas e novas, respectivamente. Deficiência hídrica reduz a colonização sobre as plantas pelos alados de B. brassicae; mas não pelos alados de $\mathbf{M}$. persicae, os quais abandonam as folhas velhas e instalam a colônia no ápice (WEARING, 1972).

KENNEDY \& MITTLER (1953) estimaram em 20 a 40 atmosferas a pressão necessária no floema para manter o fluxo normal de seiva através do canal do estilete para a alimentação de um afídio adulto. MITTLER (1957) sugere que os afídios dependem da pressão de turgescência da planta hospedeira para sua alimentação normal, sendo que essa pressão atua no sentido de forçar a ascensão da seiva no canal do estilete. AUCLAIR (1958) considera discutível que o modo de alimentação normal dos afídios seja somente por sucção. Em vista de resultados mostrando diferenças na taxa de excreção e alimentação de afídios sobre diversas variedades de ervilha, sugere que essas diferenças podem ser em parte devidas a diferenças na pressão osmótica e pressão de turgescência das diversas variedades; sendo a taxa de alimentação do afídio, até certo ponto, regulada pela planta.

TAHORI, HALEVY \& ZEIDLER (1965) verificaram que folhas de Espirradeira (Nerium oleander) tratadas com solução de cloreto de 2,4-diclorobenzil tributilfosfônico (Phosfon-D) foram menos infestadas por Aphis nerii Boyer, 1841 com relação ao controle e àquelas tratadas com cloreto de (2-cloroetil) trimetilamônio (CCC) e ácido $\mathrm{N}, \mathrm{N}$-dimetilamino succinâmico (SADH).

VAN EMDEN (1964) observou que o tratamento de Couve de Bruxelas cultivar 'Wroxton' por irrigação com solução de CCC, 15 dias após o plantio, diminuiu a longevidade, a fecundidade e a taxa de incremento de Brevicoryne brassicae.

PLAUT, HALEVY \& SHMUELI (1964) mostraram que plantas tratadas com CCC têm sua taxa de transpiração substancialmente reduzida, em presença de baixa ou alta umidade no solo. EL DAMATY, KÜHN \& LINSER (1965) concluiram que plantas de trigo tratadas com CCC, sob condições de seca utilizam sua água disponível de forma mais econômica. Plantas 
de tomateiro tratadas com $\mathrm{CCC}$ e SADH mostraram-se mais resistentes à condições de seca com relação ao controle (KNAVEL, 1969).

Traamento com ácido giberélico $\left(\mathrm{GA}_{3}\right)$ aumentou o déficit hídrico nas horas próximas do meio dia, em plantas de fumo, apesar do coeficiente transpiratório dessas plantas mostrar-se inferior ao controle (MITROFANOV, 1963).

0 presente trabalho teve como finalidade verificar a infestação de Aphis gossypii em plantas de algodoeiro cultivar 'IAC-RM3' tratadas com $\mathrm{CCC}, \mathrm{SADH}, \mathrm{GA}_{3}$ e ácido 3-indolacético (IAA), sob condições de casa de vegetação.

\section{MATERIAIS E METODOS}

Neste experimento utilizou-se o algodoeiro, Gossypium hirsutum L. cv. 'IAC-RM3'.

0 ensaio desenvolveu-se em condições de casa de vegetação, tendo-se realizado a semeadura em 22/2/74 diretamente em vasos de cerâmica com capacidade para 3 litros, contendo solo com adubo químico (N-P-K). Foram deixadas 2 plantas por vaso, efetuaram-se irrigações uniformes e não se realizou a aplicação de qualquer defensivo.

Quando as plantas apresentavam cerca de 3 folhas verdadeiras (29/3/74) ef etuou-se a aplicação de CCC (500, 1000 e 2000 ppm), SADH $(2000,3000$ e $4000 \mathrm{ppm}), \mathrm{GA}_{3}(100$ e $200 \mathrm{pm})$ e IAA $(100$ e $200 \mathrm{ppm})$. Pulve rizou-se toda a parte aérea, principalmente as folhas, até ficarem bem molhadas.

Em 29/4/74 foram conferidas notas individuais às plantas de acordo com o índice de infestação natural de A. gossypii que apresentavam; sendo que foram conferidos valores de zero (ausência de afídios) até cinco (folhas muito enrugadas). Para efeito de análise considerou-se o valor médio entre as duas plantas do vaso. Nesta mesma data foram colocados 10 afídios alados por planta; sendo que em 22/5/74 conferiram-se novas notas às plantas de acordo com a infestação agora verificada.

Durante o experimento ocorreram no local variações diárias em temperatura e umidade relativa. Registrou-se a temperatura máxima de $33,3^{\circ} \mathrm{C}$ e a mínima de $9,44^{\circ} \mathrm{C}$; a umidade relativa máxima foi da ordem de $96 \%$ e a mínima de $26 \%$.

No que se refere à análise estatística do experimento, utilizou-se o delineamento em blocos casualizados, sendo os 10 tratamentos distribuidos de forma casual em cada um dos 11 blocos, constituindo-se cada parcela de um vaso com 2 plantas. Efetuaram-se portanto as análises de variância de acordo com o esquema: 
$\begin{array}{ll}\text { Causa de variação } & \text { G.L. }\end{array}$

Blocos

Tratamentos $\quad 9$

Resíduo $\quad 90$

Total

Procedeu-se a comparação de médias pelo teste Tukey.

\section{RESULTADOS E DISCUSSÃO}

Efetuaram-se as análises dos resultados referentes às notas conferidas às plantas de acordo com o índice de infestação que apresentavam. Os valores do teste $\mathrm{F}$ bem como os coeficientes de variação dessas análises são encontrados no quadro 1.

QUADRO 1 - Efeitos da aplicação de reguladores de crescimento em 29/3/74 na infestação de plantas de algodoeiro por Aphis gossypii em 29/4/74 e 22/5/74 (valores de F para tratamentos e C.V.).

\begin{tabular}{l|c|c|r}
\hline \multicolumn{1}{c|}{ Parâmetro } & Data & F & C.V. \\
\hline Nível de & $29 / 4 / 74$ & $4,06^{* *}$ & 23,1 \\
infestação & $22 / 5 / 74$ & $7,48^{* *}$ & 15,1 \\
\hline
\end{tabular}

${ }^{* *}$ Significativo ao nível de $1 \%$

Observando-se o quadro 1 verifica-se que houve diferenças entre os tratamentos, pelo teste $F$, nas duas datas em que foram conferidas notas referentes à infestação dos afídios.

Como ocorreram diferenças entre os tratamentos, as médias, bem como as diferenças mínimas significativas determinadas pelo teste Tukey aos níveis de $5 \%$ e $1 \%$ de probabilidade foram colocadas no quadro 2. 
Quadro 2 - Medias de $\sqrt{x+0,5}$ referentes d̀s notas de dano conferidas em duas datas, de acordo com a infestação de Aphis gossypii om algodociro cultivar "IAC-RM3", e D.M.S. determinados pelo teste Tukey.

\begin{tabular}{l|l|l}
\hline \multirow{2}{*}{ Tratamentos } & \multicolumn{2}{|c}{ Médias de $\sqrt{x+0,5}$ dos valores conferidos om } \\
\cline { 2 - 3 } & $29 / 4 / 74$ & $22 / 5 / 74$ \\
\hline CCC $500 \mathrm{ppm}$ & 1,27 & 1,74 \\
CCC $1000 \mathrm{ppm}$ & 1,40 & 1,84 \\
CCC $2000 \mathrm{ppm}$ & 1,36 & 1,78 \\
SADH $2000 \mathrm{ppm}$ & 1,08 & 1,49 \\
SADH $3000 \mathrm{ppm}$ & 1,09 & 1,40 \\
SADH $4000 \mathrm{ppm}$ & 1,17 & 1,54 \\
GA $100 \mathrm{ppm}$ & 0,85 & 1,21 \\
GA $200 \mathrm{ppm}$ & 1,13 & 1,51 \\
IAA $100 \mathrm{ppm}$ & 1,03 & 1,45 \\
IAA $200 \mathrm{ppm}$ & 1,16 & 1,47 \\
\hline D.M.S. $1 \%$ & 0,43 & 0,38 \\
D.M.S. $5 \%$ & 0,37 & 0,32 \\
\hline
\end{tabular}

Verificaram-se em 29/4/74 diferenças na infestação, significativas ao nível de $1 \%$ de probabilidade entre as plantas de algodoeiro tratadas com $\mathrm{GA}_{3} 100 \mathrm{ppm}$ e aquelas tratadas com CCC $1000 \mathrm{ppm}$ e CCC $2000 \mathrm{ppm}$. Notou-se diferença ao nível de $5 \%$ de probabilidade, pelo teste Tukey, entre as plantas tratadas com $\mathrm{GA}_{3} 100 \mathrm{ppm}$ e aquelas que receberam aplicação de CCC 500 ppm (quadro 2).

Observaram-se em 22/5/74, diferenças ao nível de $1 \%$ de probabilidade na infestação dos afídios, entre o tratamento $\mathrm{GA}_{3} 100 \mathrm{ppm}$ com relação a CCC $1000 \mathrm{ppm}$, CCC $2000 \mathrm{ppm}$ e CCC $500 \mathrm{ppm}$. Verificou-se ainda diferença significativa ao nível de $5 \%$ de probabilidade, quando comparou-se $\mathrm{GA}_{3} 100$ ppm e SADH 4000 ppm (quadro 2).

Estes resultados revelam que as plantas de algodoeiro tratadas com CCC mostraram maior população de A. gossypii com relação ao tratamento com $\mathrm{GA}_{3} 100 \mathrm{ppm}$; seja pela infestação natural, seja pela infestação natural mais artificial. Apesar de não termos verificado diferença significativa entre $\mathrm{GA}_{3} 100 \mathrm{ppm}$ e $\mathrm{SADH} 4000 \mathrm{ppm}$ na primeira determinação, por ocasião da segunda observou-se diferença entre esses tratamentos.

CASTRO et al. (1976) verificaram que em folhas de regiões superiores da haste de tomateiro, o potencial osmótico mostrou-se mais elevado (menos negativo) em plantas tratadas com CCC, quando comparadas ao controle e àquelas tratadas com giberelato de potássio. 
Considerando que a dinâmica da colonização promovida pelos afídios pode estar diretamente relacionada com $o$ nível hídrico das plantas hospedeiras (WEARING, 1972), e que a taxa de alimentação dos insetos pode ser regulada pela pressão osmótica das plantas (AUCLAIR, 1958); podemos admitir que o $\mathrm{GA}_{3}$, promovendo uma diminuição no potencial osmótico com relação às plantas tratadas com CCC, possibilita um substrato alimentar menos favorável à instalação da colônia de Aphis gossypii.

Podemos considerar que as plantas tratadas com CCC suportam melhor as condições de déficits hídricos internos que ocorrem normalmente nas horas mais quentes do dia com relação àquelas tratadas com $\mathrm{GA}_{3} 100 \mathrm{ppm}$; sendo que as colônias de $A$. gossypii podem estabelecer-se ou manter-se normalmente sobre as plantas tratadas com o retardador de crescimento. Nessas plantas possivelmente o potencial osmótico é mantido mais elevado, possibilitando uma taxa mais favorável de alimentação do afídio; sendo que altas concentrações de $\mathrm{SADH}$ poderiam também possibilitar o mesmo fenômeno.

Plantas de algodoeiro cultivar 'IAC-RM3' tratadas com GA $_{3}$ na concentração de $100 \mathrm{ppm}$ devem sofrer maiores déficits hídricos internos nas horas próximas do meio dia com relação àquelas tratadas com $\mathrm{CCC}$; deste modo as colônias de $\mathbf{A}$. gossypii não têm facilidade em estabelecer-se ou manter-se nessas plantas. Nelas possivelmente o potencial osmótico é mantido mais baixo, dificultando a alimentação do afídio.

Estes resultados podem indicar a possibilidade de que plantas tratadas com CCC possam sofrer maiores infestações de afídios, possibilitando mesmo o ataque de espécies que anteriormente mostravam-se pouco importantes para a cultura por não encontrarem um substrato alimentar adequado. Porém faz-se necessário verificar se os resultados favoráveis da aplicação do regulador de crescimento na cultura não superam econômicamente estes possíveis eventos; sendo que resultados experimentais neste sentido serão de elevada importância.

\section{CONCLUSÓES}

Os resultados obtidos neste ensaio, permitem as seguintes conclusões:

1. Plantas de algodoeiro tratadas com o retardador de crescimento cloreto de (2-cloroetil) trimetilamônio, mostraram níveis de infestação de Aphis gossypii superiores com relação àquelas tratadas com ácido giberélico na concentração de $100 \mathrm{ppm}$.

2. Tratamento de plantas de algodoeiro cultivar 'IAC-RM3' com ácido $\mathrm{N}, \mathrm{N}$-dimetilamino succinâmico na concentração de $4000 \mathrm{ppm}$ revelou também uma tendência de maior infestação quando comparadas com aquelas tratadas com ácido giberélico na dosagem de $100 \mathrm{ppm}$. 


\section{SUMMARY}

\section{DIFFERENCES IN ATTACK OF Aphis gossypii ON PLANTS OF COTTON 'IAC-RM3' TREATED WITH GROWTH REGULATORS.}

We have studied the effect of application of growth regulators, (2-chloroethyl) trimethylammonium chloride (CCC), N,N-dimethylaminosuccinamic acid $(\mathrm{SADH})$, gibberellic acid $\left(\mathrm{GA}_{3}\right)$ and 3-indoleacetic acid (IAA) on cotton, Gossypium hirsutum L. cv. 'IAC-RM3', in relation to atack of Aphis gossypii Glover, 1876, under greenhouse conditions.

Two determinations of infestation levels of the aphids showed differences in degree of infestation among treatments. CCC treated plants showed increasing in aphid colonization in relation to $\mathrm{GA}_{3}$ at $100 \mathrm{ppm}$ treated plants. The application of $\mathrm{SADH}$ at $4000 \mathrm{ppm}$ also showed a tendency of increasing aphid colonization in relation to treated plants with $\mathrm{GA}_{3}$ at 100 ppm.

Probably growth retardants promote differences in the water status of hostplant; and the aphids preferences to that plants suggest that the increase in the osmotic potential promotes better rates of aphid feeding than $\mathrm{GA}_{3}$ treated plants. In treated plants with $\mathrm{GA}_{3}$ at $100 \mathrm{ppm}$ the infestation decreased, there was water stress during the warmer time of the day and a probable decreasing in the osmotic potential.

\section{AGRADECIMENTOS}

Consignamos nossos agradecimentos ao Prof. Keigo Minami do Departamento de Agricultura e Horticultura da E. S. A. «Luiz de Queiroz» - U. S. P., pelas sugestões concedidas.

\section{LITERATURA CITADA}

AUCLAIR,J. L. 1958 - Development in resistence of plants to insects. Ann. Rep. Ent. Soc. $88: 7-17$.

CALCAGNOLO, G. \& H. F. G. SAUER 1954 - A influência dc ataque dos pulgões na produção do algodão (Aphis gossypii Glover). Arq. Inst. Biol. 21 : 85-99.

CASTRO, P. R. C., M. L. R. DUARTE, C. C. MACHADO, C. CASTRO \& A. S. BRITO 1976 - Efeito de reguladores de crescimento no potencial osmótico de tomateiro (Lycopersicon esculentum Mill. cv. 'Angela'). Anais da E.S.A. «Luiz de Queiroz» (no prelo).

EL DAMATY, A . H., H. KÜHN \& H. LINSER 1965 - Water relations of wheat plants under the influence of (2-chloroethyl) - trimethyl-ammonium chloride (CCC). Physiol. Plantarum $18: 650-657$.

GALLO, D., O. NAKANO, F. M. WIENDL, S. SILVEIRA NETO \& R. P. L. CARVALHO 1970 - Manual de entomologia: pragas das plantas e seu controle. Editora Agronômica Ceres. São Paulo 1-858.

KENNEDY, J. S. 1958 - Physiological condition of the host-plant and susceptility to aphid attack. Ent. Exp. Appl. 1 : 50-65. 
KENNEDY, J. S. \& T. E. MITTLER 1953 - A method of obtaining phloem sap via the mouth-parts of aphids. Nature $171: 528$.

KNAVEL, D. E. 1969 - Influence of growth retardants on growth, nutrient content, and yield of tomato plants grown at various fertility levels. J. Amer. Soc. Hort. Sci. 94 : 32-35.

LEES, A. H. 1926 - Insect attack and the internal condition of the plant. Ann. Appl. Biol. 13 : 506-515.

MITROFANOV, B. O. 1963 - Effect of gibberellic acid on rate of photosynthesis and carbohydrate metabolism in rustic tobacco. Fisiol.-Biochim. 139-143.

MITTLER, T. E. 1957 - Studies on the feeding and nutrition of Tuberolachnus . salignus (Gmelin) (Homoptera: Aphididae). I. The uptake of phoem sap. J. Exp. Biol. $34: 334$.

PLAUT, Z., A. H. HALEVY \& SHMUELI 1964 - The effect of growth-retarding chemicals on growth and transpiration of bean plants grown under various irrigation regimes. Israel J. Agric. Res. 14 : 153-158.

TAHORI, A. S., A. H. HALEVY \& G. ZEIDLER 1965 - Effect of some plant growth retardants on the oleander aphid Aphis nerii (Boyer). J. Sci. Fd. Agric. 16 : 568-569.

VAN EMDEN, H. F. 1964 - Effect of (2-chloroethyl) trimethylammonium chloride on the rate of increase of the cabbage aphid (Brevicoryne brassicae (L.)). Nature 201 : 946-948.

WEARING, G. H. 1972 - Selection of brussels sprouts of different water status by apterous and alate Myzus persicae and Brevicoryne brassicas in relation to the age of leaves. Ent. Exp. Appl. $15: 139-154$. 